\title{
On a theorem of V. Bernik in the metric theory of Diophantine approximation
}

\author{
by \\ V. Beresnevich (Minsk)
}

1. Introduction. We begin by introducing some notation: \#S will denote the number of elements in a finite set $S$; the Lebesgue measure of a measurable set $S \subset \mathbb{R}$ will be denoted by $|S| ; P_{n}$ will be the set of integral polynomials of degree $\leq n$. Given a polynomial $P, H(P)$ will denote the height of $P$, i.e. the maximum of the absolute values of its coefficients; $P_{n}(H)=\left\{P \in P_{n}: H(P)=H\right\}$. The symbol of Vinogradov « in the expression $A \ll B$ means $A \leq C B$, where $C$ is a constant. The symbol $\asymp$ means both $\ll$ and $\gg$. Given a point $x \in \mathbb{R}$ and a set $S \subset \mathbb{R}$, let $\operatorname{dist}(x, S)=\inf \{|x-s|: x \in S\}$. Throughout, $\Psi$ will be a positive function.

K. Mahler's problem. In 1932 K. Mahler [9] introduced a classification of real numbers $x$ into the so-called classes of $A, S, T$ and $U$ numbers according to the behavior of $w_{n}(x)$ defined as the supremum of $w>0$ for which

$$
|P(x)|<H(P)^{-w}
$$

holds for infinitely many $P \in P_{n}$. By Minkowski's theorem on linear forms, one readily shows that $w_{n}(x) \geq n$ for all $x \in \mathbb{R}$. Mahler [8] has proved that for almost all $x \in \mathbb{R}$ (in the sense of Lebesgue measure), $w_{n}(x) \leq 4 n$, thus almost all $x \in \mathbb{R}$ are in the $S$-class. Mahler has also conjectured that for almost all $x \in \mathbb{R}$ one has the equality $w_{n}(x)=n$. For about 30 years the progress in Mahler's problem was limited to $n=2$ and 3 and to partial results for $n>3$. It was V. Sprindžuk who has proved Mahler's conjecture in full (see [11]).

A. Baker's conjecture. Let $W_{n}(\Psi)$ be the set of $x \in \mathbb{R}$ such that there are infinitely many $P \in P_{n}$ satisfying

$$
|P(x)|<\Psi(H(P))
$$

2000 Mathematics Subject Classification: 11J13, 11J83, 11K60.

Key words and phrases: Diophantine approximation, metric theory of Diophantine approximation, the problem of Mahler.

The work has been supported by EPSRC grant GR/R90727/01. 
A. Baker [1] has improved Sprindžuk's theorem by showing that

$$
\left|W_{n}(\Psi)\right|=0 \text { if } \sum_{h=1}^{\infty} \Psi^{1 / n}(h)<\infty \text { and } \Psi \text { is monotonic. }
$$

He has also conjectured a stronger statement, proved over 20 years later by V. Bernik [5], that $\left|W_{n}(\Psi)\right|=0$ if the sum

$$
\sum_{h=1}^{\infty} h^{n-1} \Psi(h)
$$

converges and $\Psi$ is monotonic. Afterwards V. Beresnevich [2] has shown that $\left|\mathbb{R} \backslash W_{n}(\Psi)\right|=0$ if (2) diverges and $\Psi$ is monotonic. Here we prove

Theorem 1. Let $\Psi: \mathbb{R} \rightarrow \mathbb{R}^{+}$be an arbitrary function (not necessarily monotonic) such that the sum (2) converges. Then $\left|W_{n}(\Psi)\right|=0$.

Theorem 1 is no longer improvable as, by [2], the convergence of (2) is crucial. Notice that for $n=1$ the theorem is simple and known (see, for example, [7, p. 121]). Therefore, from now on we assume that $n \geq 2$.

2. Subcases of Theorem 1. Let $\delta>0$. We define the following three sets $W_{\mathrm{big}}(\Psi), W_{\text {med }}(\Psi)$ and $W_{\text {small }}(\Psi)$ consisting of $x \in \mathbb{R}$ such that there are infinitely many $P \in P_{n}$ simultaneously satisfying (1) and one of the following inequalities:

$$
\begin{aligned}
1 & \leq\left|P^{\prime}(x)\right|, \\
H(P)^{-\delta} & \leq\left|P^{\prime}(x)\right|<1, \\
\left|P^{\prime}(x)\right| & <H(P)^{-\delta},
\end{aligned}
$$

respectively. Obviously $W_{n}(\Psi)=W_{\text {big }}(\Psi) \cup W_{\text {med }}(\Psi) \cup W_{\text {small }}(\Psi)$. Hence to prove Theorem 1 it suffices to show that each of these sets has zero measure.

Since the sum (2) converges, $H^{n-1} \Psi(H)$ tends to 0 as $H \rightarrow \infty$. Therefore,

$$
\Psi(H)=o\left(H^{-n+1}\right) \quad \text { as } H \rightarrow \infty .
$$

3. The case of a big derivative. The aim of this section is to prove that $\left|W_{\text {big }}(\Psi)\right|=0$. Let $B_{n}(H)$ be the set of $x \in \mathbb{R}$ such that there exists a polynomial $P \in P_{n}(H)$ satisfying (3). Then

$$
W_{\text {big }}(\Psi)=\bigcap_{N=1}^{\infty} \bigcup_{H=N}^{\infty} B_{n}(H) .
$$

Now $\left|W_{\text {big }}(\Psi)\right|=0$ if $\left|W_{\text {big }}(\Psi) \cap I\right|=0$ for any open interval $I \subset \mathbb{R}$ satisfying

$$
0<c_{0}(I)=\inf \{|x|: x \in I\}<\sup \{|x|: x \in I\}=c_{1}(I)<\infty .
$$

Therefore we can fix an interval $I$ satisfying (8). 
By (7) and the Borel-Cantelli Lemma, $\left|W_{\text {big }}(\Psi) \cap I\right|=0$ whenever

$$
\sum_{H=1}^{\infty}\left|B_{n}(H) \cap I\right|<\infty .
$$

By the convergence of (2), condition (9) will follow on showing that

$$
\left|B_{n}(H) \cap I\right| \ll H^{n-1} \Psi(H)
$$

with the implicit constant in (10) independent of $H$.

Given a $P \in P_{n}(H)$, let $\sigma(P)$ be the set of $x \in I$ satisfying (3). Then

$$
B_{n}(H) \cap I=\bigcup_{P \in P_{n}(H)} \sigma(P) .
$$

Lemma 1. Let $I$ be an interval with endpoints $a$ and $b$. Define $I^{\prime \prime}=$ $[a, a+4 \Psi(H)] \cup[b-4 \Psi(H), b]$ and $I^{\prime}=I \backslash I^{\prime \prime}$. Then for all sufficiently large $H$, for any $P \in P_{n}(H)$ such that $\sigma(P) \cap I^{\prime} \neq \emptyset$, for any $x_{0} \in \sigma(P) \cap I^{\prime}$ there exists $\alpha \in I$ such that $P(\alpha)=0,\left|P^{\prime}(\alpha)\right|>\left|P^{\prime}\left(x_{0}\right)\right| / 2$ and $\left|x_{0}-\alpha\right|<$ $2 \Psi(H)\left|P^{\prime}(\alpha)\right|^{-1}$.

The proof of this lemma nearly coincides with the one of Lemma 1 in [2] and is left to the reader. There will be some changes to constants and notation and one will also have to use (6).

Given a polynomial $P \in P_{n}(H)$ and a real number $\alpha$ such that $P^{\prime}(\alpha) \neq 0$, define $\sigma(P ; \alpha)=\left\{x \in I:|x-\alpha|<2 \Psi(H)\left|P^{\prime}(\alpha)\right|^{-1}\right\}$. Let $I^{\prime}$ and $I^{\prime \prime}$ be as in Lemma 1. For every polynomial $P \in P_{n}(H)$, we define the set

$$
Z_{I}(P)=\left\{\alpha \in I: P(\alpha)=0 \text { and }\left|P^{\prime}(\alpha)\right| \geq 1 / 2\right\} .
$$

By Lemma 1 , for any $P \in P_{n}(H)$ we have the inclusion

$$
\sigma(P) \cap I^{\prime} \subset \bigcup_{\alpha \in Z_{I}(P)} \sigma(P ; \alpha) .
$$

Given $k \in \mathbb{Z}$ with $0 \leq k \leq n$, define

$$
P_{n}(H, k)=\left\{P=a_{n} x^{n}+\cdots+a_{0} \in P_{n}(H): a_{k}=0\right\}
$$

and for $R \in P_{n}(H, k)$ let

$$
P_{n}(H, k, R)=\left\{P \in P_{n}(H): P-R=a_{k} x^{k}\right\} .
$$

It is easily observed that

$$
P_{n}(H)=\bigcup_{k=0}^{n} \bigcup_{R \in P_{n}(H, k)} P_{n}(H, k, R)
$$

and

$$
\# P_{n}(H, k) \ll H^{n-1} \quad \text { for every } k .
$$

Taking into account (11), (13), (14) and the fact that $\left|I^{\prime \prime}\right| \ll \Psi(H)$, it now becomes clear that to prove (10) it is sufficient to show that for every fixed 
$k$ and fixed $R \in P_{n}(H, k)$,

$$
\left|\bigcup_{P \in P_{n}(H, k, R)} \sigma(P) \cap I^{\prime}\right| \ll \Psi(H) .
$$

Let $k$ and $R$ be fixed. Define the rational function $\widetilde{R}(x)=x^{-k} R(x)$. By (8), there exists a collection of intervals $\left[w_{i-1}, w_{i}\right) \subset I(i=1, \ldots, s)$, which do not intersect pairwise and cover $I$, such that $\widetilde{R}^{\prime}(x)$ is monotonic and does not change sign on every interval $\left[w_{i-1}, w_{i}\right)$. It is clear that $s$ depends on $n$ only. Let $Z_{I, R}=\bigcup_{P \in P_{n}(H, k, R)} Z_{I}(P), k_{i}=\#\left(Z_{I, R} \cap\left[w_{i-1}, w_{i}\right)\right)$ and $Z_{I, R} \cap\left[w_{i-1}, w_{i}\right)=\left\{\alpha_{i}^{(1)}, \ldots, \alpha_{i}^{\left(k_{i}\right)}\right\}$, where $\alpha_{i}^{(j)}<\alpha_{i}^{(j+1)}$. Given a $P \in$ $P_{n}(H, k, R)$, we obviously have the identity

$$
\frac{x^{k} P^{\prime}(x)-k x^{k-1} P(x)}{x^{2 k}}=\left(\frac{P(x)}{x^{k}}\right)^{\prime}=\widetilde{R}^{\prime}(x) .
$$

Taking $x$ to be $\alpha \in Z_{I}(P)$ leads to $P^{\prime}(\alpha) / \alpha^{k}=\widetilde{R}^{\prime}(\alpha)$. By $(8),\left|P^{\prime}(\alpha)\right| \asymp$ $\left|\widetilde{R}^{\prime}(\alpha)\right|$. Now, by Lemma $1,|\sigma(P ; \alpha)| \ll \Psi(H)\left|P^{\prime}(\alpha)\right|^{-1} \ll \Psi(H)\left|\widetilde{R}^{\prime}(\alpha)\right|^{-1}$.

Using (12), we get

$$
\left|\bigcup_{P \in P_{n}(H, k, R)} \sigma(P) \cap I^{\prime}\right| \ll \Psi(H) \sum_{i=1}^{s} \sum_{j=1}^{k_{i}} \frac{1}{\left|\widetilde{R}\left(\alpha_{i}^{(j)}\right)\right|} .
$$

Now to show (15) it suffices to prove that for every $i(1 \leq i \leq s)$,

$$
\sum_{j=1}^{k_{i}}\left|\widetilde{R}^{\prime}\left(\alpha_{i}^{(j)}\right)\right|^{-1} \ll 1
$$

Fix an index $i(1 \leq i \leq s)$. If $k_{i} \geq 2$ then we can consider two sequential roots $\alpha_{i}^{(j)}$ and $\alpha_{i}^{(j+1)}$ of two rational functions $\widetilde{R}+a_{k}^{i, j}$ and $\widetilde{R}+a_{k}^{i, j+1}$ respectively. For convenience let us assume that $\widetilde{R}^{\prime}$ is increasing and positive on $\left[w_{i-1}, w_{i}\right)$. Then $\widetilde{R}$ is strictly monotonic on $\left[w_{i-1}, w_{i}\right)$, and we have $a_{k}^{i, j} \neq a_{k}^{i, j+1}$. It follows that $\left|a_{k}^{i, j}-a_{k}^{i, j+1}\right| \geq 1$. Using the Mean Value Theorem and the monotonicity of $\widetilde{R}^{\prime}$, we get

$$
\begin{aligned}
1 & \leq\left|a_{0}^{i, j}-a_{0}^{i, j+1}\right|=\left|\widetilde{R}^{\prime}\left(\alpha_{i}^{(j)}\right)-\widetilde{R}^{\prime}\left(\alpha_{i}^{(j+1)}\right)\right|=\left|\widetilde{R}^{\prime}\left(\widetilde{\alpha}_{i}^{(j)}\right)\right| \cdot\left|\alpha_{i}^{(j)}-\alpha_{i}^{(j+1)}\right| \\
& \leq\left|\widetilde{R}^{\prime}\left(\alpha_{i}^{(j+1)}\right)\right| \cdot\left|\alpha_{i}^{(j)}-\alpha_{i}^{(j+1)}\right|,
\end{aligned}
$$

where $\widetilde{\alpha}_{i}^{(j)}$ is a point between $\alpha_{i}^{(j)}$ and $\alpha_{i}^{(j+1)}$. This implies $\left|\widetilde{R}^{\prime}\left(\alpha_{i}^{(j+1)}\right)\right|^{-1} \leq$ $\alpha_{i}^{(j+1)}-\alpha_{i}^{(j)}$, whence we readily get

$$
\sum_{j=1}^{k_{i}-1}\left|\widetilde{R}^{\prime}\left(\alpha_{i}^{(j+1)}\right)\right|^{-1} \leq \sum_{j=1}^{k_{i}-1}\left(\alpha_{i}^{(j+1)}-\alpha_{i}^{(j)}\right)=\alpha_{i}^{\left(k_{i}\right)}-\alpha_{i}^{(1)} \leq w_{i}-w_{i-1} .
$$


The last inequality and $\left|\widetilde{R}^{\prime}\left(\alpha_{i}^{(1)}\right)\right| \asymp\left|P^{\prime}\left(\alpha_{i}^{(1)}\right)\right| \gg 1$ yield (16). It is easily verified that (16) holds for every $i$ with $k_{i} \geq 2$ and is certainly true when $k_{i}=1$ or $k_{i}=0$. This completes the proof of the case of a big derivative.

4. The case of a medium derivative. As above we fix an interval $I$ satisfying (8). Then $\left|W_{\text {med }}(\Psi)\right|=0$ will follow from $\left|W_{\text {med }}(\Psi) \cap I\right|=0$. We will use the following

Lemma 2 (see Lemma 2 in [3]). Let $\alpha_{0}, \ldots, \alpha_{k-1}, \beta_{1}, \ldots, \beta_{k} \in \mathbb{R} \cup\{\infty\}$ be such that $\alpha_{0}>0, \alpha_{j}>\beta_{j} \geq 0$ for $j=1, \ldots, k-1$ and $0<\beta_{k}<\infty$. Let $f:(a, b) \rightarrow \mathbb{R}$ be a $C^{(k)}$ function such that $\inf _{x \in(a, b)}\left|f^{(k)}(x)\right| \geq \beta_{k}$. Then the set of $x \in(a, b)$ satisfying

$$
|f(x)| \leq \alpha_{0}, \quad \beta_{j} \leq\left|f^{(j)}(x)\right| \leq \alpha_{j} \quad(j=1, \ldots, k-1)
$$

is a union of at most $k(k+1) / 2+1$ intervals, each with length at most $\min _{0 \leq i<j \leq k} 3^{(j-i+1) / 2}\left(\alpha_{i} / \beta_{j}\right)^{1 /(j-i)}$. Here we assume $\frac{c}{0}=\infty$ for $c>0$.

Given a polynomial $P \in P_{n}(H)$, we redefine $\sigma(P)$ to be the set of solutions of (4). Since $P^{(n)}(x)=n ! a_{n}$, we can apply Lemma 2 to $P$ with $k=n$ and

$$
\begin{gathered}
\alpha_{0}=\Psi(H), \quad \alpha_{1}=1, \quad \beta_{1}=\inf _{x \in \sigma(P)}\left|P^{\prime}(x)\right| \geq H^{-\delta}, \quad \beta_{n}=1, \\
\alpha_{2}=\cdots=\alpha_{n-1}=\infty, \quad \beta_{2}=\cdots=\beta_{n-1}=0 .
\end{gathered}
$$

Then we conclude that $\sigma(P)$ is a union of at most $n(n+1) / 2+1$ intervals of length $\ll \alpha_{0} / \beta_{1}$. There is no loss of generality in assuming that the sets $\sigma(P)$ are intervals, as otherwise, we would treat the intervals of $\sigma(P)$ separately. We can also ignore those $P$ for which $\sigma(P)$ is empty. For every $P$ we define a point $\gamma_{P} \in \sigma(P)$ such that $\inf _{x \in \sigma(P)}\left|P^{\prime}(x)\right| \geq \frac{1}{2}\left|P^{\prime}\left(\gamma_{P}\right)\right|$. The existence is easily seen. Now we have

$$
|\sigma(P)| \ll \Psi(H)\left|P^{\prime}\left(\gamma_{P}\right)\right|^{-1} .
$$

It also follows from the choice of $\gamma_{P}$ that

$$
H(P)^{-\delta} \leq\left|P^{\prime}\left(\gamma_{P}\right)\right|<1 .
$$

Now define expansions of $\sigma(P)$ as follows:

$$
\begin{aligned}
& \sigma_{1}(P):=\left\{x \in I: \operatorname{dist}(x, \sigma(P))<\left(H\left|P^{\prime}\left(\gamma_{P}\right)\right|\right)^{-1}\right\}, \\
& \sigma_{2}(P):=\left\{x \in I: \operatorname{dist}(x, \sigma(P))<H^{-1+2 \delta}\right\} .
\end{aligned}
$$

By (4), $\sigma_{1}(P) \subset \sigma_{2}(P)$. Moreover, it is easy to see that

$$
\sigma_{1}(P) \subset \sigma_{2}(Q) \quad \text { for any } Q \in P_{n}(H) \text { with } \sigma_{1}(Q) \cap \sigma_{1}(P) \neq \emptyset \text {. }
$$

It is also readily verified that $\left|\sigma_{1}(P)\right| \asymp\left(H\left|P^{\prime}\left(\gamma_{P}\right)\right|\right)^{-1}$, and therefore, by $(17)$,

$$
|\sigma(P)| \ll\left|\sigma_{1}(P)\right| H \Psi(H)
$$


Take any $x \in \sigma_{2}(P)$. Using the Mean Value Theorem, (18) and the fact that $\left|x-\gamma_{P}\right| \ll H^{-1+2 \delta}$, we get

$$
\left|P^{\prime}(x)\right| \leq\left|P^{\prime}\left(\gamma_{P}\right)\right|+\left|P^{\prime \prime}(\widetilde{x})\left(x-\gamma_{P}\right)\right| \ll 1+H \cdot H^{-1+2 \delta} \ll H^{2 \delta},
$$

where $\widetilde{x}$ is between $x$ and $\gamma_{P}$. Similarly we estimate $|P(x)|$ :

$$
|P(x)| \ll H^{-1+4 \delta}, \quad\left|P^{\prime}(x)\right| \ll H^{2 \delta} \quad \text { for any } x \in \sigma_{2}(P) .
$$

Now for every pair $(k, m)$ of integers with $0 \leq k<m \leq n$ we define

$$
P_{n}(H, k, m)=\left\{R=a_{n} x^{n}+\cdots+a_{0} \in P_{n}(H): a_{k}=a_{m}=0\right\}
$$

and for a given polynomial $R \in P_{n}(H, k, m)$ we define

$$
P_{n}(H, k, m, R)=\left\{P=R+a_{m} x^{m}+a_{k} x^{k} \in P_{n}(H)\right\} .
$$

The intervals $\sigma(P)$ will be divided into 2 classes of essential and nonessential intervals. The interval $\sigma(P)$ will be essential if for any choice of $(k, m, R)$ such that $P \in P_{n}(H, k, m, R)$ for any $Q \in P_{n}(H, k, m, R)$ other than $P$ we have $\sigma_{1}(P) \cap \sigma_{1}(Q)=\emptyset$. For fixed $k, m$ and $R$ summing the measures of essential intervals gives

$$
\sum|\sigma(P)| \leq H \Psi(H) \sum\left|\sigma_{1}(P)\right| \leq H \Psi(H)|I| \ll H \Psi(H) .
$$

As $\# P_{n}(H, k, m) \ll H^{n-2}$ and there are only $n(n+1) / 2$ different pairs $(k, m)$ we obtain the following estimate:

$$
\sum_{\text {essential intervals } \sigma(P)}|\sigma(P)| \ll H^{n-1} \Psi(H) .
$$

Thus, by the Borel-Cantelli Lemma and the convergence of (2), the set of points $x$ of $W_{\text {med }}(\Psi) \cap I$ which belong to infinitely many essential intervals is of measure zero.

Now let $\sigma(P)$ be non-essential. Then, by definition and (19), there is a choice of $k, m, R$ such that $P \in P_{n}(H, k, m, R)$ and there is a $Q \in$ $P_{n}(H, k, m, R)$ different from $P$ such that

$$
\sigma(P) \subset \sigma_{1}(P) \subset \sigma_{2}(P) \cap \sigma_{2}(Q) .
$$

On the set $\sigma_{2}(P) \cap \sigma_{2}(Q)$ both $P$ and $Q$ satisfy (20) and so does the difference $P(x)-Q(x)=b_{m} x^{m}+b_{k} x^{k}$. It is not difficult to see that $b_{m} \neq 0$ if $H$ is large enough. Therefore using (20) we get

$$
\left|x^{m-k}+\frac{b_{k}}{b_{m}}\right| \ll \frac{H^{-1+4 \delta}}{\left|b_{m}\right|} \leq H^{-1+4 \delta}, \quad \max \left\{\left|b_{m}\right|,\left|b_{k}\right|\right\} \ll H^{2 \delta} .
$$

Now let $x$ belong to infinitely many non-essential intervals. Without loss of generality we assume that $x$ is transcendental as otherwise it belongs to a countable set, which is of measure zero. Therefore (21) is satisfied for 
infinitely many $b_{m}, b_{k} \in \mathbb{Z}$. Hence, the inequality

$$
\left|x^{m-k}-\frac{p}{q}\right|<q^{-(1-5 \delta) / 2 \delta}
$$

holds for infinitely many $p, q \in \mathbb{Z}$. Taking $\delta=1 / 10$ so that $(1-5 \delta) / 2 \delta$ becomes $2+\delta$, and applying standard Borel-Cantelli arguments (see [7, p. 121]) we complete the proof of the case of a medium derivative for nonessential intervals.

5. The case of a small derivative. In this section we prove that $\left|W_{\text {small }}(\Psi)\right|=0$. We will make use of Theorem 1.4 in [6]. By taking $d=1$, $\mathbf{f}=\left(x, x^{2}, \ldots, x^{n}\right), U=\mathbb{R}, T_{1}=\cdots=T_{n}=H, \theta=H^{-n+1}, K=H^{-\delta}$ in that theorem, we arrive at

Theorem 2. Let $x_{0} \in \mathbb{R}$ and

$$
\delta^{\prime}=\frac{\min (\delta, n-1)}{(n+1)(2 n-1)} .
$$

Then there exists a finite interval $I_{0} \subset \mathbb{R}$ containing $x_{0}$ and a constant $E>0$ such that

$$
\left|\bigcup_{P \in P_{n}, 0<H(P) \leq H}\left\{x \in I_{0}:|P(x)|<H^{-n+1},\left|P^{\prime}(x)\right|<H^{-\delta}\right\}\right| \leq E H^{-\delta^{\prime}} .
$$

In particular Theorem 2 implies that, for any $\delta>0$, the set of $x \in \mathbb{R}$ for which there are infinitely many polynomials $P \in P_{n}$ satisfying the system

$$
|P(x)|<H(P)^{-n+1}, \quad\left|P^{\prime}(x)\right|<H(P)^{-\delta},
$$

has zero measure. Indeed, this set consists of points $x \in I_{0}$ which belong to infinitely many sets

$\tau_{m}=\left\{x \in I_{0}:(22)\right.$ holds for some $P \in P_{n}$ with $\left.2^{m-1}<H(P) \leq 2^{m}\right\}$.

By Theorem 2, $\left|\tau_{m}\right| \ll 2^{-m \delta^{\prime}}$ with $\delta^{\prime}>0$. Therefore, $\sum_{m=1}^{\infty}\left|\tau_{m}\right|<\infty$ and the Borel-Cantelli Lemma completes the proof of the claim.

In view of (6), this completes the proof of the case of a small derivative and the proof of Theorem 1 .

6. Concluding remarks. An analogue of Theorem 1 when $P$ is assumed to be irreducible over $\mathbb{Q}$ and primitive (i.e. with coprime coefficients) can also be sought. To make it more precise, let $P_{n}^{*}(H)$ be the subset of $P_{n}(H)$ consisting of primitive irreducible polynomials $P$ of degree $\operatorname{deg} P=n$ and height $H(P)=H$. Now the set of primitive irreducible polynomials of degree $n$ is $P_{n}^{*}=\bigcup_{H=1}^{\infty} P_{n}^{*}(H)$. Let $W_{n}^{*}(\Psi)$ be the set of $x \in \mathbb{R}$ such that there are infinitely many $P \in P^{*}$ satisfying (1). 
TheOREm 3. Let $\Psi: \mathbb{R} \rightarrow \mathbb{R}^{+}$be an arbitrary function such that the sum

$$
\sum_{H=1}^{\infty} \frac{\# P_{n}^{*}(H)}{H} \Psi(H)
$$

converges. Then $\left|W_{n}^{*}(\Psi)\right|=0$.

For $n=1$ the proof of Theorem 3 is a straightforward application of the Borel-Cantelli Lemma and we again refer to [7, p. 121]. For $n>1$ the proof is deduced from the following two observations: 1) $W_{n}^{*}(\Psi) \subset W_{n}(\Psi)$ and 2) $\# P_{n}^{*}(H) \asymp H^{n}$. The second one guarantees the convergence of (2), which now implies $0 \leq\left|W_{n}^{*}(\Psi)\right| \leq\left|W_{n}(\Psi)\right|=0$. The proof of the relation $\# P_{n}^{*}(H) \asymp H^{n}$ is elementary and is left to the reader. In fact, one can easily estimate the number of primitive reducible polynomials in $P_{n}$ and take them off the set of all primitive polynomials in $P_{n}$ which is well known to contain at least a constant times $\# P_{n}(H)$ elements.

The Duffin-Schaeffer conjecture. The conjecture states that for $n=1$ if (23) diverges then $\left|\mathbb{R} \backslash W_{n}^{*}(\Psi)\right|=0$. The multiple $\# P_{1}^{*}(H)$ in (23) becomes $\asymp \varphi(H)$, where $\varphi$ is the Euler function.

The following problem can be regarded as the generalisation of the Duffin-Schaeffer conjecture to integral polynomials of higher degree:

Prove that $\left|\mathbb{R} \backslash W_{n}^{*}(\Psi)\right|=0$ whenever (23) diverges.

Alternatively, for $n>1$ one might investigate the measure of $\mathbb{R} \backslash W_{n}(\Psi)$. So far it is unclear if for $n>1,\left|\mathbb{R} \backslash W_{n}^{*}(\Psi)\right|=0$ is equivalent to $\left|\mathbb{R} \backslash W_{n}(\Psi)\right|$ $=0$, which is another intricate question.

A remark on manifolds. In the metric theory of Diophantine approximation on manifolds one usually studies sets of $\Psi$-approximable points lying on a manifold with respect to the measure induced on that manifold. Mahler's problem and its generalisations can be regarded as Diophantine approximation on the Veronese curve $\left(x, x^{2}, \ldots, x^{n}\right)$.

A point $\mathbf{f} \in \mathbb{R}^{n}$ is called $\Psi$-approximable if

$$
\|\mathbf{a} \cdot \mathbf{f}\|<\Psi\left(|\mathbf{a}|_{\infty}\right)
$$

for infinitely many $\mathbf{a} \in \mathbb{Z}^{n}$, where $|\mathbf{a}|_{\infty}=\max _{1 \leq i \leq n}\left|a_{i}\right|$ for $\mathbf{a}=\left(a_{1}, \ldots, a_{n}\right)$, $\|x\|=\min \{|x-z|: z \in \mathbb{Z}\}$ and $\Psi: \mathbb{R} \rightarrow \mathbb{R}^{+}$.

Let $\mathbf{f}: U \rightarrow \mathbb{R}^{n}$ be a map defined on an open set $U \subset \mathbb{R}^{d}$. We say that $\mathbf{f}$ is non-degenerate at $\mathbf{x}_{0} \in U$ if for some $l \in \mathbb{N}$ the map $\mathbf{f}$ is $l$ times continuously differentiable on a sufficiently small ball centered at $\mathbf{x}_{0}$ and there are $n$ linearly independent over $\mathbb{R}$ partial derivatives of $\mathbf{f}$ at $\mathbf{x}_{0}$ of orders up to $l$. We say that $\mathbf{f}$ is non-degenerate if it is non-degenerate almost everywhere on $U$. The non-degeneracy of a manifold is naturally defined via the non-degeneracy of its local parameterisation. 
In 1998 D. Kleinbock and G. Margulis proved the Baker-Sprindžuk conjecture by showing that any non-degenerate manifold is strongly extremal. In particular, this implies an analogue of Mahler's problem for non-degenerate manifolds. A few years later an analogue of A. Baker's conjecture with monotonic $\Psi$ (normally called a Groshev type theorem for convergence) has independently been proven by V. Beresnevich [3] and by V. Bernik, D. Kleinbock and G. Margulis [6] for non-degenerate manifolds. It is also remarkable that the proofs were given with different methods. The divergence counterpart (also for monotonic $\Psi$ ) has been established in [4]. In [6] a multiplicative version of the Groshev type theorem for convergence has also been given.

Theorem 1 of this paper can be readily generalised to non-degenerate curves: Given a non-degenerate map $\mathbf{f}: I \rightarrow \mathbb{R}^{n}$ defined on an interval $I$, for any function $\Psi: \mathbb{R} \rightarrow \mathbb{R}^{+}$such that the sum (2) converges for almost all $x \in I$ the point $\mathbf{f}(x)$ is not $\Psi$-approximable. Even further, using the slicing technique of Pyartli [10] one can extend this to a class of $n$-differentiable non-degenerate manifolds which can be foliated by non-degenerate curves. In particular, this class includes arbitrary non-degenerate analytic manifolds. However with the technique at our disposal we are currently unable to prove the following

ConjeCture. Let $\mathbf{f}: U \rightarrow \mathbb{R}^{n}$ be a non-degenerate map, where $U$ is an open subset of $\mathbb{R}^{d}$. Then for any function $\Psi: \mathbb{R} \rightarrow \mathbb{R}^{+}$such that the sum (2) converges for almost all $\mathbf{x} \in U$ the point $\mathbf{f}(\mathbf{x})$ is not $\Psi$-approximable.

\section{References}

[1] A. Baker, On a theorem of Sprindžuk, Proc. Roy. Soc. London Ser. A 292 (1966), 92-104.

[2] V. V. Beresnevich, On approximation of real numbers by real algebraic numbers, Acta Arith. 90 (1999), 97-112.

[3] - , A Groshev type theorem for convergence on manifolds, Acta Math. Hungar. 94 (2002), 99-130.

[4] V. V. Beresnevich, V. I. Bernik, D. Y. Kleinbock and G. A. Margulis, Metric Diophantine approximation: the Khintchine-Groshev theorem for nondegenerate manifolds, Moscow Math. J. 2 (2002), 203-225.

[5] V. I. Bernik, On the exact order of approximation of zero by values of integral polynomials, Acta Arith. 53 (1989), 17-28 (in Russian).

[6] V. I. Bernik, D. Y. Kleinbock and G. A. Margulis, Khintchine-type theorems on manifolds: the convergence case for standard and multiplicative versions, Internat. Math. Res. Notices 2001, no. 9, 453-486.

[7] J. W. S. Cassels, An Introduction to Diophantine Approximation, Cambridge Univ. Press, Cambridge, 1957.

[8] K. Mahler, Über das Maß der Menge aller S-Zahlen, Math. Ann. 106 (1932), 131139. 
[9] K. Mahler, Zur Approximation der Exponentialfunktion und des Logarithmus, I, II, J. Reine Angew. Math. 166 (1932), 118-136, 137-150.

[10] A. S. Pyartli, Diophantine approximations on submanifolds of Euclidean space, Funkts. Anal. i Prilozhen. 3 (1969), no. 4, 59-62 (in Russian); English transl.: Funct. Anal. Appl. 3 (1970), 303-306.

[11] V. G. Sprindžuk, Mahler's Problem in Metric Number Theory, Transl. Math. Monogr. 25, Amer. Math. Soc., Providence, RI, 1969.

Institute of Mathematics

Academy of Sciences of Belarus

220072, Surganova 11, Minsk, Belarus

E-mail: beresnevich@im.bas-net.by

Received on 3.6.2004

and in revised form on 24.10.2004 